\title{
Development of Multifunctional CNTs Reinforced PEI Filaments for Fused Deposition Modeling
}

\author{
Özge Kaynan ${ }^{1}$, Alptekin Yıldız², Yunus Emre Bozkurt ${ }^{3}$, Elif Özden Yenigün ${ }^{4}$, and Hülya Cebeci ${ }^{5}$ \\ Aerospace Research Center, Istanbul Technical University, Istanbul, Turkey, 34469 \\ School of Design, Textiles, Royal College of Art, London, United Kingdom, SW7 2EU
}

\begin{abstract}
Additive manufacturing (AM) enables cost effective production of complex shapes with providing design freedom. Fused deposition modeling (FDM) has been one of the most accessible AM methods which guide thermoplastic filaments to provide accurate and easy production of 3D objects layer by layer fusion. However, this technique has brought some drawbacks associated with limited material choices including relatively weak structural properties, low resolution range, and restrained processability in 3D printers. To overcome these flaws of FDM, herein we described the fabrication of high-performance thermoplastic filaments as an FDM feedstock as a stronger replacement of commodity thermoplastics. For further improvement, carbon nanotubes (CNTs) were incorporated into high performance matrices to provide multifunctionality both by improving mechanical properties and electrical conductivity. To achieve that, composite polyetherimide (PEI) filaments with various CNTs fractions were processed by melt compounding without any solvents or additives. Manufacturing process adopted a sequence of twin and single screw extrusion. Thermal transition and rheological changes due to CNTs incorporation were monitored and morphology, tensile behavior and electrical conductivity of neat PEI and nanocomposite filaments were investigated. The results showed that $5 \mathrm{wt} \% \mathrm{CNTs}$ reinforced PEI filaments exhibited $55 \%$ higher stiffness compared to neat PEI feedstock. Structural analysis supported that these nanofillers were well dispersed in mix state and electrical percolation threshold of CNTs/PEI nanocomposite filaments was found as low as ca. $0.1 \mathrm{wt} \%$ CNTs.
\end{abstract}

\section{Introduction}

A bility to develop complex structures and intricate components with minimum waste, design freedom and mass customization are offered by 3D printing as a manufacturing revolution [1]. One of the most widely used additive manufacturing techniques, fused deposition modeling (FDM), provides accurate and easy production of 3D objects through layer by layer fusion. Limitations arising from 3D printers such as just building prototype quality parts, low resolution range between 50-200 $\mu \mathrm{m}$, and print size restrictions are being matured to fabricate end-user products in several fields. However, from a materials perspective, a limited number of suitable thermoplastic filaments is also under intense attention to create multifunctional feedstocks that can be used in customized 3D printers for applications in aerospace, automotive, digital art and design, and architecture [2].

To date, open source 3D printers use commodity thermoplastics such as polylactic acid (PLA) and acrylonitrile butadiene styrene (ABS) feedstocks with insufficient mechanical properties including poor strength and elongation. Instead of using common thermoplastics as FDM feedstocks, 3D parts fabricated by high-performance filaments offer high stiffness and strength, which becomes promising to use as load-bearing components for advanced applications. High-performance filaments to be extruded in expensive FDM printers such as polyetheretherketone (PEEK), polyetherimide (PEI), polysulfone (PS) and polyphenylsulfone (PPS) are already commercially available in the market $[3,4]$.

Particularly PEI as a melt processable, amorphous polymer exhibits outstanding mechanical and chemical properties with very high thermal resistance and dimensional stability for aerospace applications [5]. Most importantly with a specific strength of $85 \mathrm{kN} \cdot \mathrm{m} / \mathrm{kg}$ compared to stainless steel with $60 \mathrm{kN} \cdot \mathrm{m} / \mathrm{kg}, \mathrm{PEI}$ is a good alternative to replace

${ }^{1} \mathrm{PhD}$ Student, Department of Textile Engineering, Istanbul Technical University, Turkey

${ }^{2} \mathrm{PhD}$ Candidate, Department of Physics Engineering, Istanbul Technical University, Turkey

${ }^{3} \mathrm{MSc}$ Student, Department of Defense Technologies, Istanbul Technical University, Turkey

${ }^{4}$ Senior Tutor, Department of Textiles, Royal College of Art, UK, Corresponding Author: elif.ozden-yenigun@ @rca.ac.uk

${ }^{5}$ Associate Professor, Department of Aeronautical Engineering, Istanbul Technical University, Turkey, AIAA Member, Corresponding Author: hulya.cebeci@itu.edu.tr 
metal use in parts and components when strength-weight ratio is considered. Recent efforts focused on the addition of conductive nanofillers into FDM filament that provides multifunctionality to the feedstock and later to 3D printed structures through enhancing mechanical performance and electrical conductivity without significant weight penalty $[6,7]$.These nanofillers could be metallic or carbon-based materials such as graphite, graphene and carbon nanotubes (CNTs). CNTs are rolled graphite sheet with a diameter of a few nanometers and a few micrometers length, which have an excellent reinforcing capability within their outstanding aspect ratio and mechanical properties.

In this study, PEI filaments with various CNTs fractions were fabricated by melt compounding to fabricate $\mathrm{CNT}$ reinforced PEI nanocomposite filaments (CNTs/PEI) for FDM. Morphology, tensile behavior, and electrical conductivity of fabricated composite filaments were investigated. PEI filaments with CNTs weight fractions ranging from 0.1 to $7 \mathrm{wt}$ $\%$ were fabricated by melt compounding as FDM feedstock without any solvents or additives by a customized twin and single screw extruders as seen in Figure 1. To fabricate CNTs/PEI nanocomposite filaments first, PEI granules were dried in a vacuum oven for 8 hour (h) to remove moisture content and then, neat PEI granules were fed into the main feeder of twin screw extrusion. Meanwhile, CNTs were fed into the twin screw extruder through side feeder which is closer to the die. Twin screw extrusion process was applied for five times for each batch to ensure homogeneous dispersion and distribution of CNTs into PEI matrix. Following to twin screw extrusion, CNTs/PEI nanocomposite pellets were obtained by a granulator. The nanocomposite pellets, then, were fed through the main hopper of single screw extruder to fabricate CNTs/PEI nanocomposite filaments with a standard diameter. CNTs dispersion and distribution were investigated by scanning electron microscopy (SEM) and transmission electron microscopy (TEM), and thermal stability and degradation characteristics were determined by thermogravimetric analysis (TGA). Mechanical properties of the neat PEI and CNT/PEI nanocomposite filaments were characterized by tensile testing and electrical conductivity of the filaments was measured by a four-point probe measuring system.

a)

b)
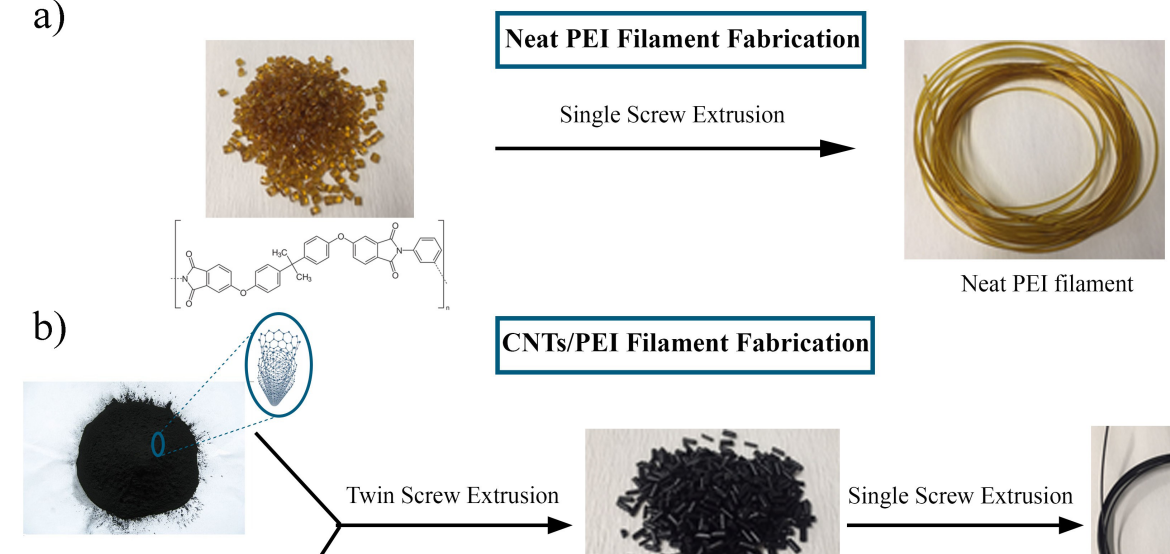

Neat PEI filament

CNTs/PEI Filament Fabrication

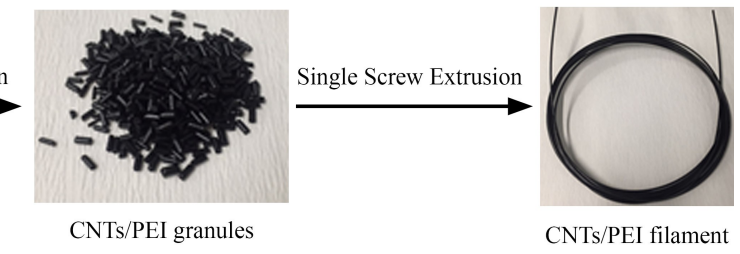

Single Screw Extruder

c) Twin Screw Extruder

(2) (3) (4) (5) (6)

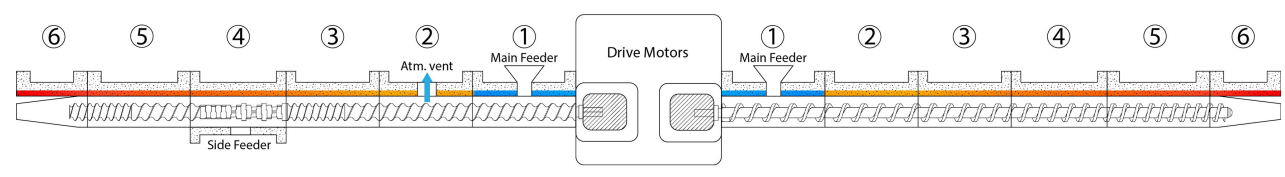

Fig. 1 Manufacturing processes for a) neat and b) CNTs/PEI filaments by melt compounding, and c) a schematic describing custom made twin and single screw extruder configurations. 


\section{Experimental Approach}

\section{A. Materials}

Polyetherimide pellets were purchased from Eurotech (ULTEM 1000, MW: 54000). CNTs as reinforcing material were purchased from Nanokomp (industrial scale multi walled CNTs (MWCNTs), carbon purity: min. $90 \%$, diameter: 5-15 nm, length: $1.5-2 \mu \mathrm{m})$. All materials were used without any purification and neat and nanocomposite filaments were manufactured without using any additives.

\section{B. Fabrication of PEI and CNTs/PEI Nanocomposite Filament}

Before melt compounding process, first neat PEI granules were dried at $150^{\circ} \mathrm{C}$ for $8 \mathrm{~h}$ to remove moisture absorbed in the polymer. Neat PEI filaments were fabricated by single screw extruder at $60 \mathrm{rpm}$ screw speed with a drawing ratio of 20. Afterwards, co-rotating twin screw extruder and single screw extruder (screw diameter of $12 \mathrm{~mm}$, length and diameter ratio (L/D) of 22 for both twin and single screw extruders) were used in sequence to achieve proper melt compounding and filament production.

For nanocomposite filament production, CNTs at different mass fractions were blended into PEI matrix using twin screw extruder. 2, 5 and $7 \mathrm{wt} \% \mathrm{CNTs} / \mathrm{PEI}$ nanocomposite pellets were fabricated using this method. To fabricate lower mass fraction of CNT/PEI nanocomposite pellets, $2 \mathrm{wt} \% \mathrm{CNTs} / \mathrm{PEI}$ pellets were used as base and diluted to achieve $0.1,0.25$ and 0.5 wt \% CNTs/PEI pellets. Further, each type of pellets whether neat or/and nanocomposite PEI granules were fed to single screw extruder by $60 \mathrm{rpm}$ screw speed with a drawing ratio of 20 . The barrel temperatures were kept at $30,310,330,340,350$, and $360^{\circ} \mathrm{C}$, as illustrated in Figure 1c, Zones from 1 to 6, respectively.

\section{Characterization}

\section{Thermogravimetric analysis}

The thermal stability of neat PEI and CNTs/PEI nanocomposites were tested by thermogravimetric analysis (TGA). The analysis was carried out using TGA 8000 Perkin Elmer instrument at heating rate of $10^{\circ} \mathrm{C} / \mathrm{min}$, under inert $\mathrm{N}_{2}$ atmosphere and the samples were heated from room temperature to $800^{\circ} \mathrm{C}$ with a gas purge at $20 \mathrm{ml} / \mathrm{min}$. Neat PEI, 5 and 7 wt \% CNTs/PEI nanocomposites were analyzed to determine the effect of CNTs on thermal stability and decomposition temperature.

\section{Rheological analysis}

The effect of CNTs on polymer flow was studied by using a rheometer (Anton Paar MCR 102). Prior to testing, each group of granules was conditioned in a vacuum oven for $4 \mathrm{~h}$. The sample holder of rheometer was made of disposable aluminum with $25 \mathrm{~mm}$ diameter and $2 \mathrm{~mm}$ height. The samples were placed into the holder, and kept at $360^{\circ} \mathrm{C}$ for 5 minutes to be melted. Angular frequency tests were performed in the range of 0.1 and $500 \mathrm{rad} / \mathrm{sec}$.

\section{Morphological investigations}

The surface morphologies of neat PEI and CNTs/PEI nanocomposites were observed using field emission scanning electron microscope (FE-SEM). SEM analysis were performed by using SEM, LEO 1530VP operating at $15 \mathrm{keV}$. Prior to SEM measurements, specimens were fractured in cryogenic liquid and coated with thin layer of gold-platinum alloy. Thereafter, samples were placed in a vacuum chamber in order to focalize electron beam on the fractured edge. Transmission electron microscope (TEM) analysis was carried out using a JEOL JEM-ARM200CFEG UHR-TEM device operated at $200 \mathrm{kV}$. Thin slides of nanocomposites having a thickness of $100 \mathrm{~nm}$ were prepared by JEOL JIB-4601F MultiBeam focused ion beam (FIB) to obtain quantitative measures of CNTs size, their distribution, and dispersion state.

\section{Electrical conductivity measurements}

The electrical conductivity of neat PEI and CNTs/PEI nanocomposites was evaluated by four-point probe measuring system FPP 470 (Entek Electronics, Turkey) at room temperature. Each filament with $2.5 \mathrm{~cm}$ length and $0.85 \mathrm{~mm}$ thickness was prepared and then polished to reduce surface roughness. Four samples of each material composition were tested at their geometrically center points. Electrical conductivity evaluations were performed according to calculation 
method described in [8].

\section{Tensile properties of filaments}

The tensile properties of neat PEI and $5 \mathrm{wt} \% \mathrm{CNTs} / \mathrm{PEI}$ filaments were tested at room temperature by Zwick Roell Z100 testing machine with a load capacity of $100 \mathrm{kN}$, in which single bollard self-tightening type grids with manual extensometer was mounted. Test procedures were followed based on ASTM 3822D standard. Filaments with diameter of $1.75 \mathrm{~mm}$ cut into $60 \mathrm{~cm}$ length, $110 \mathrm{~mm}$ gauge length were set in testing. Each specimen was tested at least five times.

\section{Result and Discussion}

\section{A. Thermal Properties}

Thermal degradation properties of neat and nanocomposite granules, and the effect of CNTs on thermal transitions were studied as illustrated in Figure 2. Neat PEI granules showed the characteristic two step thermal decomposition. First transition at $c a .500^{\circ} \mathrm{C}$ was related to the decomposition of non-aromatic chemical structure of PEI, and the second one seen at $c a .550^{\circ} \mathrm{C}$ was due to decomposition of aromatic groups [9]. Figure 2 pointed out that the degradation temperatures of neat PEI, 5 and $7 \mathrm{wt} \% \mathrm{CNTs} / \mathrm{PEI}$ filaments were 556,555 and $551^{\circ} \mathrm{C}$, respectively. Overall, it was suggested that CNTs addition had minimal impact on degradation temperature of PEI, which could be also interpreted as having small effect on processability during 3D printing process. However, the effect of CNTs addition became apparent when calculating residual amounts at higher temperatures. Weight loss of neat PEI, 5 and $7 \mathrm{wt} \%$ CNTs reinforced PEI were $45 \%, 37 \%$ and $36 \%$, respectively at $800^{\circ} \mathrm{C}$. This result suggests that as detected at high temperatures, CNTs could have improve thermal stability compared to neat PEI due to dispersed CNTs' physical barrier in matrix.

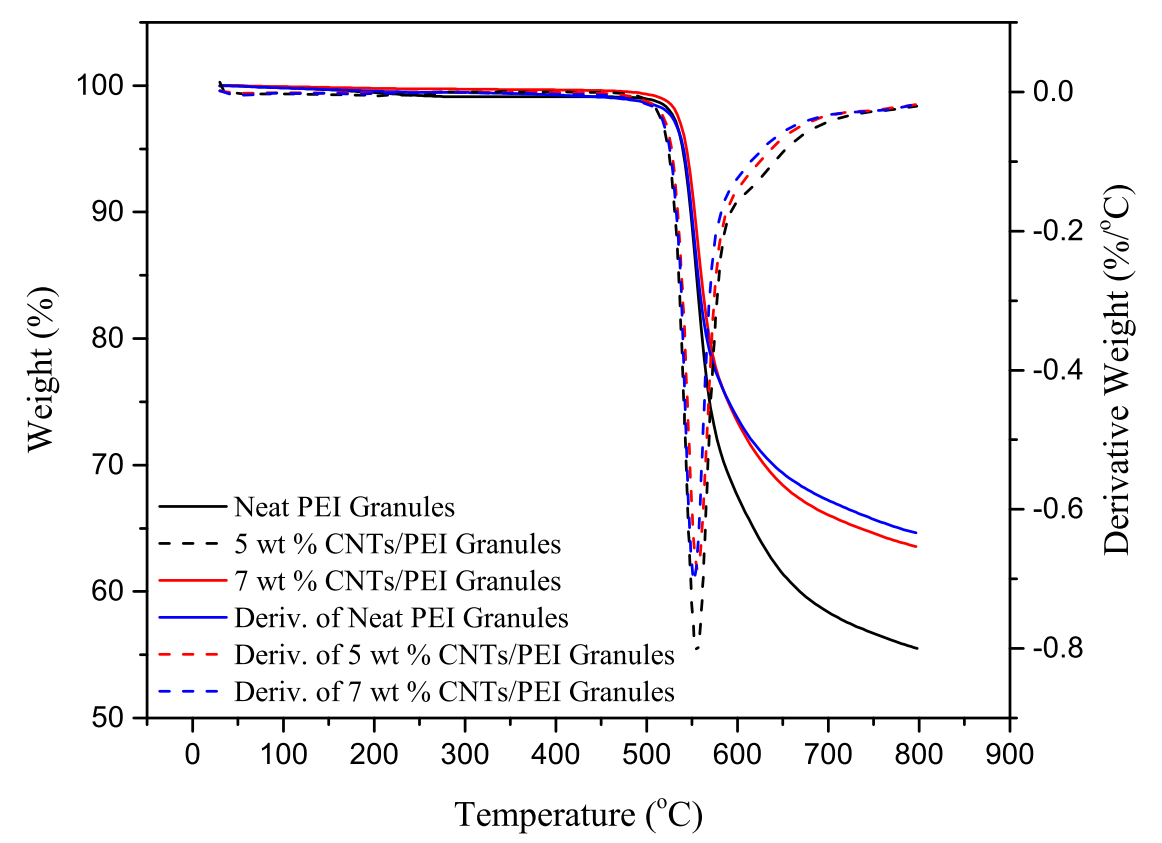

Fig. 2 Thermogravimetric analysis of neat PEI, 5 wt \% and $7 \mathrm{wt} \% \mathrm{CNTs} / \mathrm{PEI}$ granules 


\section{B. Rheology}

Figure 3 shows the complex viscosity as a function of frequency for neat PEI, 0.1, 0.25 and 5 wt $\%$ CNTs/PEI granules. In contrast to some polymers such as PEEK [10], neat PEI showed frequency dependency that was observed by shear thinning behavior with increasing frequency. Moreover, melt viscosity of neat PEI was measured around $5 \times 10^{5} \mathrm{~Pa} \cdot \mathrm{s}$, which was much higher than other high-performance polymers such as PEEK [10] and HDPE [11] This also highlighted well-known difficulties in melt processing of PEI. In the measurements, 0.1 and 0.5 wt \% CNTs/PEI filaments also showed similar characteristics as neat PEI due to low CNTs weight fractions. The difference between 5 wt $\% \mathrm{CNTs} / \mathrm{PEI}$ and PEI with low fraction of CNTs was particularly obvious at low frequencies. This difference diminished due to strong shear thinning effect when the frequency was increased. This could be attributed to dispersive forces which helped dispersing CNTs in melted PEI matrix and/or effective interfacial strength between the nanofiller and the matrix $[12,13]$.

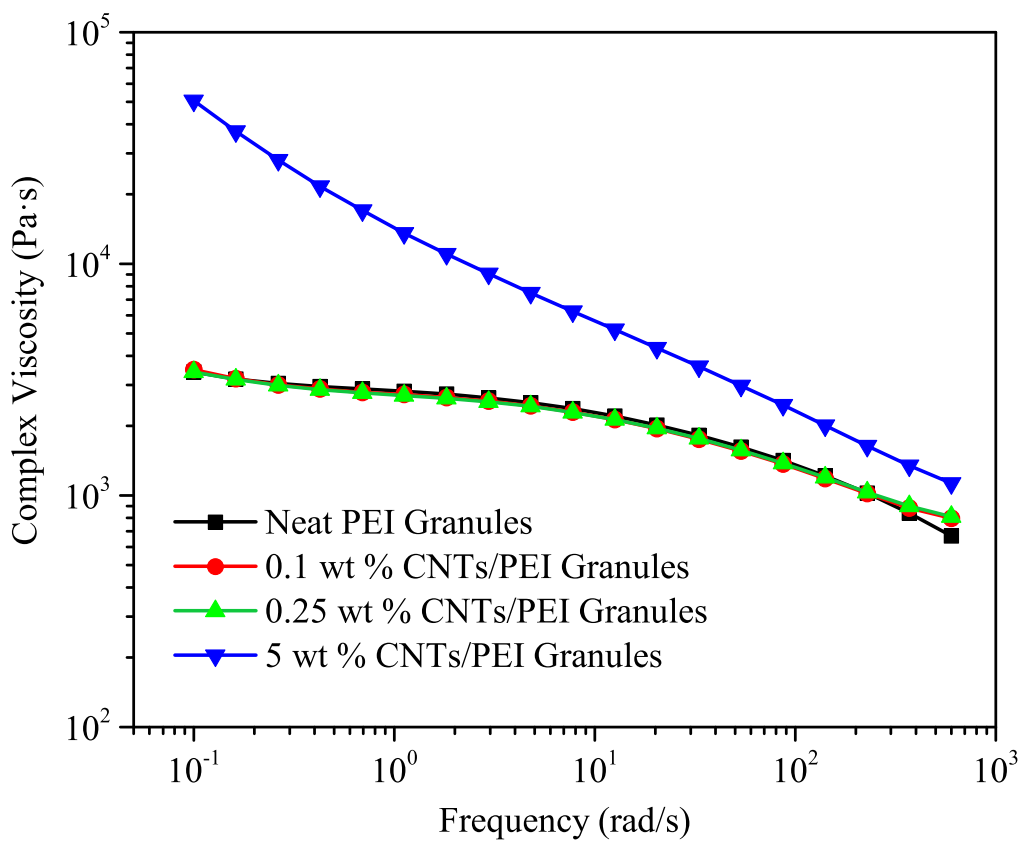

Fig. 3 Thermogravimetric analysis of neat PEI, 5 wt \% and 7 wt \% CNTs/PEI granules

\section{Morphological Properties}

Figure 4 reveals cross-section morphologies of neat PEI, 5 and 7 wt \% CNTs/PEI nanocomposite filaments. As seen in Figure 4a, PEI surface was smooth where sharp cracks were visible attributed to uninterrupted breakage of filament. Figure $4 \mathrm{~b}$ and $4 \mathrm{c}$ exhibited rougher surfaces taken from 5 and $7 \mathrm{wt} \% \mathrm{CNTs} / \mathrm{PEI}$ filaments, respectively. The fibrillary formation seen in the images were identified as CNTs tails immersed in polymer matrix [7]. These complimentary figures demonstrate that CNTs were homogeneously dispersed in PEI matrix due to a sequence of twin screw extrusion process. Furthermore, CNTs were well adhered to PEI matrix which was a consequence of high compatibility between CNTs and PEI.

Much clearer observation of CNTs dispersion in PEI matrix was provided through TEM images as in Figure 5. TEM analysis revealed that CNTs were very well dispersed and distributed in PEI matrix even at high CNTs loadings of $7 \mathrm{wt} \%$. Moreover, CNTs were also aligned through extrusion direction revealing effective dispersion attributed to using both single and twin screw extruders for mixing. Compared to nanocomposites' TEM images of reference studies $[14,15]$ fabricated by solely twin screw extruder, in which nanofiller agglomerates were detected clearly proving the importance of processing conditions. After the successful dispersion and distribution process, re-agglomeration of 


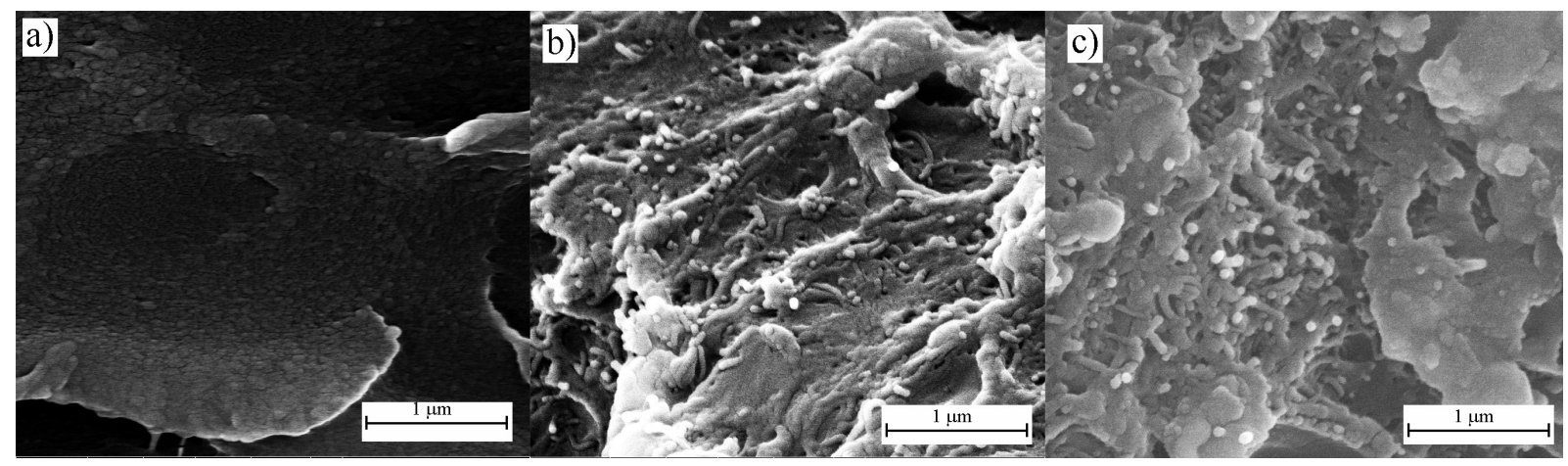

Fig. 4 SEM images of a) neat PEI, b) $5 \mathrm{wt} \%$ CNTs/PEI and c) $7 \mathrm{wt} \%$ CNTs/PEI filaments.

CNTs was not observed due to high viscosity of PEI. Hence, it is also noteworthy that PEI was a good host for CNTs [14]. As a drawback of extrusion process, applied high shear stress during nanocomposite fabrication was expected in reduction of CNTs lengths. The original length of CNTs was reported as 1.5-2 $\mu \mathrm{m}$ through data sheet however, after the extrusion process, the average of CNTs lengths was reduced to $305 \pm 20 \mathrm{~nm}$.

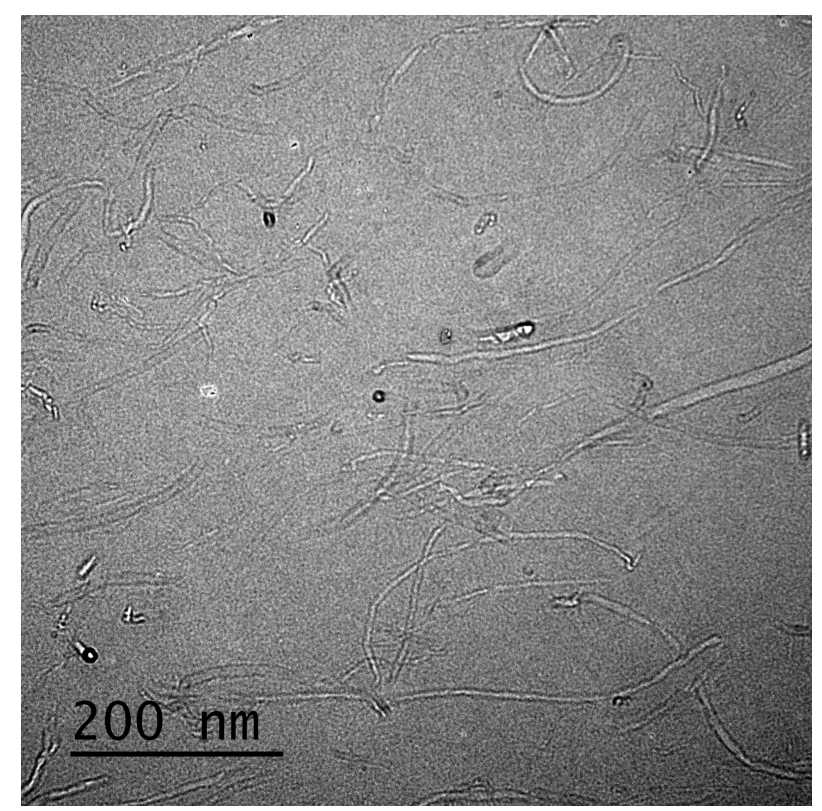

Fig. 5 TEM image of $7 \mathrm{wt} \%$ CNTs/PEI filaments.

\section{Tensile Testing}

Tensile behavior of neat PEI and 5 wt \% CNTs/PEI nanocomposite filaments was demonstrated in Figure 6. The tensile strength of PEI filaments remained almost the same even with the addition of CNTs, which were $93 \pm 1.7 \mathrm{MPa}$ and $87 \pm 3 \mathrm{MPa}$ for neat PEI and $5 \mathrm{wt} \% \mathrm{CNTs} / \mathrm{PEI}$ nanocomposites, respectively. However, the elongation at break $\%$ of neat PEI filament reduced from $20 \pm 4 \%$ to $7 \pm 3 \%$ due to high CNTs loading. Significant improvement in stiffness was observed, and $5 \% \mathrm{wt}$. of CNT addition had increased the modulus from $2.13 \pm 0.6 \mathrm{GPa}$ to $3.31 \pm 0.13 \mathrm{GPa}$ compared to PEI matrix. The effect of CNTs addition on tensile modulus was directly dependent on the success of dispersion, and proper aspect ratio of CNTs and degree of alignment caused through extrusion process [5]. As revealed in TEM images in Figure 5, aspect ratio of CNTs was severely affected from high shear stress applied during twin screw extrusion process. Hence, interfacial shear strength between PEI and CNTs, dispersion and alignment of CNTs through the filament axis could have contributed to enhancement of tensile modulus. 


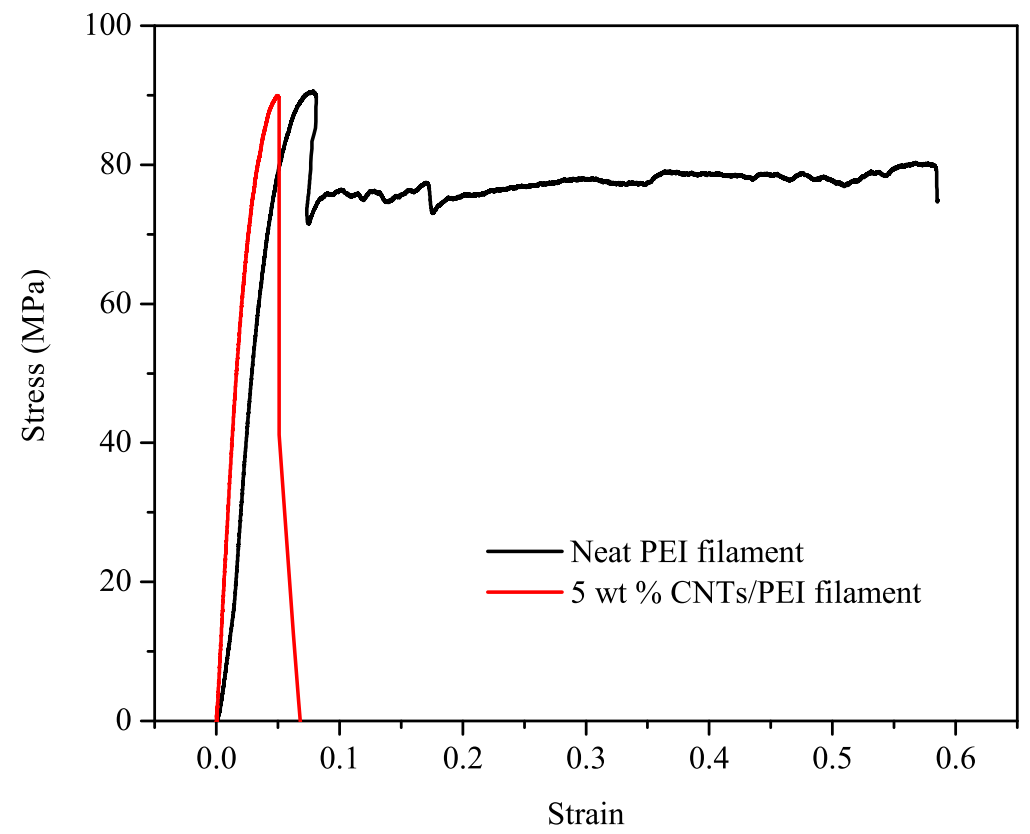

Fig. 6 Tensile properties of neat PEI and 5 wt \% CNTs/PEI filaments.

\section{E. Electrical Conductivity}

Surface conductivity of neat PEI and CNTs/PEI nanocomposite filaments were represented in Table 1. The electrical conductivity of filaments was drastically enhanced with increasing CNTs weight fraction as also observed by in reference study [14]. Isayev et al. [16] reported electrical conductivity of $10 \mathrm{wt} \%$ CNTs/PEI nanocomposites produced by ultrasonically assisted twin screw extruder as around $10^{-5} \mathrm{~S} / \mathrm{cm}$. In this study, percolation threshold of CNTs/PEI nanocomposite filaments were found between 0.1 and $0.25 \mathrm{wt} \% \mathrm{CNTs}$, which was clearly lower than other reported CNTs nanocomposites such as CNTs/PLA (0.67 wt \%) [17] and CNTs/PBI (0.49 wt \%) [18]. The main reason to succeed lower percolation threshold compared to earlier studies on thermoplastics was relied on creating a conductive network with improved homogeneous dispersion of CNTs, in spite of lower aspect ratio of CNTs. Also, low percolation threshold of CNTs/PEI filaments indicated that CNTs did not agglomerate in the PEI matrix as also revealed in TEM images and a conductive network was created throughout the filament [19]. Percolation threshold succeeded at low CNTs fraction also pointed out CNTs did not re-agglomerate due to high viscous PEI matrix.

Table 1 Surface conductivity measurements of CNTs/PEI filaments at different wt \% CNTs

\begin{tabular}{cc}
\hline CNTs wt \% in PEI matrix & Surface Conductivity (S/cm) \\
\hline 0 & $10^{-15}$ \\
0.1 & $1.25 \times 10^{-5}$ \\
0.25 & $1.67 \times 10^{-3}$ \\
0.5 & $1.77 \times 10^{-3}$ \\
2 & $4.23 \times 10^{-3}$ \\
5 & $1.69 \times 10^{-1}$ \\
7 & $2.57 \times 10^{-1}$ \\
\hline
\end{tabular}




\section{F. Preliminary Printed Samples}

Figure 7 demonstrates the 3D printed dog-bone samples and various geometries fabricated by using neat PEI and 5 wt \% CNTs/PEI filaments.

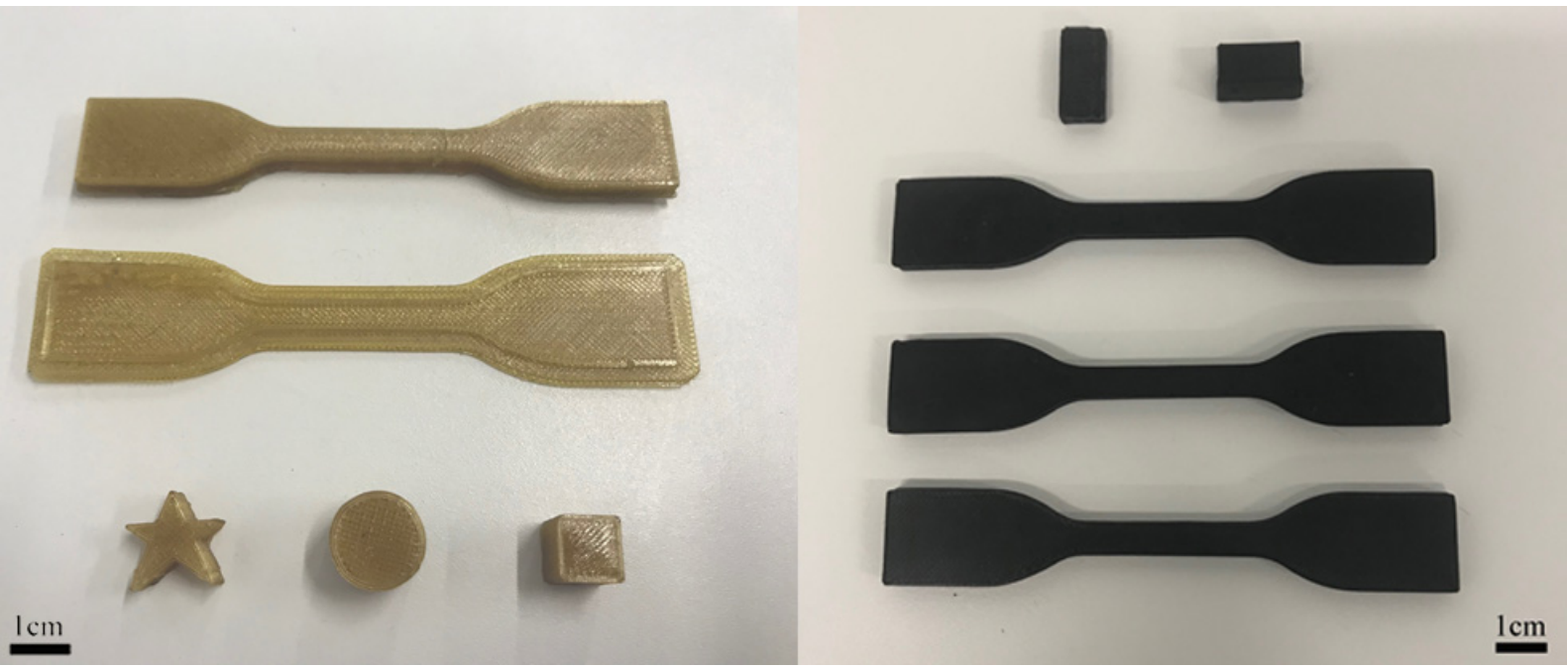

Fig. 7 3D printed dog-bone and samples with different geometries produced by using of neat PEI (left) and 5 wt \% CNTs/PEI (right) filaments.

\section{Conclusion}

In conclusion, CNTs/PEI nanocomposite filaments with various weight fractions of CNTs were fabricated by consecutive twin and single screw extrusion process for later use in additive manufacturing enabling multifunctional 3D structures. The sequence of twin and single screw extrusions in CNTs/PEI nanocomposite filament fabrication resulted in uniform dispersion and alignment of CNTs in PEI matrix, which contributed positively to both tensile performance and percolation threshold even at low nanofiller content. However, reduction in CNTs length was also observed due to applied high shear stress during melt compounding. Despite this depletion in CNTs aspect ratio, CNTs presence led to increase in stiffness up to $55 \%$ because of efficient interfacial strength, dispersion and alignment in PEI matrix. Nevertheless, reduction in elongation at break \%, which was around $35 \%$, was noted as negative contribution of CNTs on brittleness. The results suggested that surface conductivity of PEI was markedly enhanced by CNTs incorporation up to $2.57 \times 10^{-1} \mathrm{~S} / \mathrm{cm}$.

\section{Acknowledgement}

The authors thank to the Boeing Global Co. for equipment support, TUBITAK Project Numbered (116M427) for graduate student scholarship support of Özge Kaynan, and ITU BAP Project Numbered (2017-40710) for material support.

\section{References}

[1] Ngo, T. D., Kashani, A., Imbalzano, G., Nguyen, K. T., and Hui, D., "Additive manufacturing (3D printing): A review of materials, methods, applications and challenges," Composites Part B: Engineering, Vol. 143, 2018, pp. 172-196. doi: 10.1016/j.compositesb.2018.02.012.

[2] Wang, X., Jiang, M., Zhou, Z., Gou, J., and Hui, D., “3D printing of polymer matrix composites: A review and prospective," Composites Part B: Engineering, Vol. 110, 2017, pp. 442-458. doi:10.1016/j.compositesb.2016.11.034.

[3] "Apium,", 2018. URL https://apiumtec.com.

[4] “3DXTech,”, 2018. URL https://www.3dxtech.com. 
[5] Pitchan, M. K., Bhowmik, S., Balachandran, M., and Abraham, M., "Effect of surface functionalization on mechanical properties and decomposition kinetics of high performance polyetherimide/MWCNT nano composites," Composites Part A: Applied Science and Manufacturing, Vol. 90, 2016, pp. 147-160. doi:10.1016/j.compositesa.2016.06.025.

[6] Zhang, D., Chi, B., Li, B., Gao, Z., Du, Y., Guo, J., and Wei, J., "Fabrication of highly conductive graphene flexible circuits by 3D printing," Synthetic Metals, Vol. 217, 2016, pp. 79-86. doi:10.1016/j.synthmet.2016.03.014.

[7] Dul, S., Fambri, L., and Pegoretti, A., "Filaments production and fused deposition modelling of ABS/carbon nanotubes composites," Nanomaterials, Vol. 8, No. 1, 2018, p. 49. doi:10.3390/nano8010049.

[8] Miccoli, I., Edler, F., Pfnür, H., and Tegenkamp, C., "The 100th anniversary of the four-point probe technique: the role of probe geometries in isotropic and anisotropic systems," Journal of Physics: Condensed Matter, Vol. 27, No. 22, 2015, p. 223201. doi:10.1088/0953-8984/27/22/223201.

[9] Abbasi, H., Antunes, M., and Velasco, J., "Graphene nanoplatelets-reinforced polyetherimide foams prepared by water vapor-induced phase separation.” eXPRESS Polymer Letters, Vol. 9, No. 5, 2015. doi:10.3144/expresspolymlett.2015.40.

[10] Bangarusampath, D., Ruckdäschel, H., Altstädt, V., Sandler, J. K., Garray, D., and Shaffer, M. S., "Rheology and properties of melt-processed poly(ether ether ketone)/multi-wall carbon nanotube composites," Polymer, Vol. 50, No. 24, 2009, pp. 5803-5811. doi:10.1016/j.polymer.2009.09.061.

[11] Lotti, C., Isaac, C. S., Branciforti, M. C., Alves, R. M., Liberman, S., and Bretas, R. E., "Rheological, mechanical and transport properties of blown films of high density polyethylene nanocomposites," European Polymer Journal, Vol. 44, No. 5, 2008, pp. 1346-1357. doi:10.1016/j.eurpolymj.2008.02.014.

[12] Zhong, J., and Isayev, A. I., "Properties of polyetherimide/graphite composites prepared using ultrasonic twin-screw extrusion," Journal of Applied Polymer Science, Vol. 132, No. 5, 2015. doi:10.1002/app.41397.

[13] Li, B., Olson, E., Perugini, A., and Zhong, W.-H., "Simultaneous enhancements in damping and static dissipation capability of polyetherimide composites with organosilane surface modified graphene nanoplatelets," Polymer, Vol. 52, No. 24, 2011, pp. 5606-5614. doi:10.1016/j.polymer.2011.09.048.

[14] Socher, R., Krause, B., Müller, M. T., Boldt, R., and Pötschke, P., “The influence of matrix viscosity on MWCNT dispersion and electrical properties in different thermoplastic nanocomposites," Polymer, Vol. 53, No. 2, 2012, pp. 495-504. doi: 10.1016/j.polymer.2011.12.019.

[15] Berretta, S., Davies, R., Shyng, Y. T., Wang, Y., and Ghita, O., "Fused Deposition Modelling of high temperature polymers: Exploring CNT PEEK composites," Polymer Testing, Vol. 63, 2017, pp. 251-262. doi:10.1016/j.polymertesting.2017.08.024.

[16] Isayev, A., Kumar, R., and Lewis, T. M., "Ultrasound assisted twin screw extrusion of polymer-nanocomposites containing carbon nanotubes,” Polymer, Vol. 50, No. 1, 2009, pp. 250-260. doi:10.1016/j.polymer.2008.10.052.

[17] Postiglione, G., Natale, G., Griffini, G., Levi, M., and Turri, S., "Conductive 3D microstructures by direct 3D printing of polymer/carbon nanotube nanocomposites via liquid deposition modeling," Composites Part A: Applied Science and Manufacturing, Vol. 76, 2015, pp. 110-114. doi:10.1016/j.compositesa.2015.05.014.

[18] Gnanasekaran, K., Heijmans, T., van Bennekom, S., Woldhuis, H., Wijnia, S., de With, G., and Friedrich, H., "3D printing of CNT- and graphene-based conductive polymer nanocomposites by fused deposition modeling," Applied Materials Today, Vol. 9, 2017, pp. 21-28. doi:10.1016/j.apmt.2017.04.003.

[19] Li, J., Ma, P. C., Chow, W. S., To, C. K., Tang, B. Z., and Kim, J. K., "Correlations between percolation threshold, dispersion state, and aspect ratio of carbon nanotubes," Advanced Functional Materials, Vol. 17, No. 16, 2007, pp. 3207-3215. doi: 10.1002/adfm.200700065. 